PROCEEDINGS OF THE

AMERICAN MATHEMATICAL SOCIETY

Volume 132, Number 7 , Pages 2051-2055

S 0002-9939(03)07295-2

Article electronically published on December 23, 2003

\title{
A BOUND FOR THE DIMENSION OF THE AUTOMORPHISM GROUP OF A HOMOGENEOUS COMPACT COMPLEX MANIFOLD
}

\author{
DENNIS M. SNOW
}

(Communicated by Richard A. Wentworth)

\begin{abstract}
Let $X$ be a homogeneous compact complex manifold, and let Aut $(X)$ be the complex Lie group of holomorphic automorphisms of $X$. Examples show that $\operatorname{dim} \operatorname{Aut}(X)$ can grow exponentially in $n=\operatorname{dim} X$. In this note it is shown that

$$
\operatorname{dim} \operatorname{Aut}(X) \leq n^{2}-1+\left(\begin{array}{c}
2 n-1 \\
n-1
\end{array}\right)
$$

when $n \geq 3$. Thus, $\operatorname{dim} \operatorname{Aut}(X)$ is at most exponential in $n$. The proof relies on an upper bound for the dimension of the space of sections of the anticanonical bundle, $K_{Y}^{*}=\operatorname{det} T_{Y}$, of a homogeneous projective rational manifold $Y$ of dimension $m: \operatorname{dim} H^{0}\left(Y, K_{Y}^{*}\right) \leq\left(\begin{array}{c}2 m+1 \\ m\end{array}\right)$.
\end{abstract}

\section{INTRODUCTION}

Let $X$ be a connected compact complex manifold. Then $\operatorname{Aut}(X)$ is a complex Lie group acting holomorphically on $X$ 2. If $X$ is homogeneous under $\operatorname{Aut}(X)$, we may identify $X$ with a coset space $G / H$ where $G=\operatorname{Aut}^{0}(X)$ is the connected component of the identity of $\operatorname{Aut}(X)$ and $H$ is the closed complex subgroup fixing a point in $X$. One simple measure of the degree of homogeneity of $X$ is given by the dimension of its automorphism group, $d=\operatorname{dim} \operatorname{Aut}(X)$. It is natural to ask how large $d$ can be relative to the dimension $n$ of $X$ and to seek those homogeneous compact complex manifolds $X$ of a fixed dimension $n$ for which $d$ is a maximum.

If $X$ can be equivariantly embedded in complex projective space, then standard arguments involving Lie's theorem imply that the radical of $G$ acts trivially on $X$ and hence $G$ is semisimple. Moreover, a maximal compact subgroup $K \subset G$ acts transitively, $X=K / L, L=K \cap H$, and the isotropy representation of $L$ is an embedding into the unitary group $U(n)$. Therefore, $d=\operatorname{dim}_{\mathbb{C}} G=\operatorname{dim}_{\mathbb{R}} K=$ $2 n+\operatorname{dim}_{\mathbb{R}} L \leq 2 n+n^{2}$. More generally, if $X=G / H$ is Kähler, then $X=Y \times Z$ where $Y$ is homogeneous and admits an equivariant embedding into projective space, and $Z$ is a compact complex torus [3]. It follows that the same estimate on $d$ holds: $d \leq n(n+2)$. Furthermore, it is not hard to verify that equality occurs only in the case where $X$ is a complex projective space, $X=\mathbb{P}^{n}$. Thus, the question about the maximum of $d$ for a fixed $n$ is completely answered in the Kähler case.

Received by the editors November 10, 2002 and, in revised form, March 20, 2003.

2000 Mathematics Subject Classification. Primary 32M10; Secondary 32M05. 
The non-Kähler case does not yet have such complete answers. It has been known for some time that for each $n$ there is a theoretical upper bound for $d$; see [1, p. 99]. Examples show that an upper bound for $d$ must be at least exponential in $n$ [7]. The construction of these examples relies on several delicately balanced factors, including the existence of certain uniform discrete subgroups, which make it seem unlikely that a precise maximum $d$ will be found for each $n$. Nevertheless, in this note we establish the following explicit estimate for $d$ when $n \geq 3$ :

$$
d \leq n^{2}-1+\left(\begin{array}{c}
2 n-1 \\
n-1
\end{array}\right) .
$$

Stirling's formula reveals that $\left(\begin{array}{c}2 n-1 \\ n-1\end{array}\right)$ asymptotically approaches $2^{2 n-1} / \sqrt{(n-1) \pi}$. Therefore, $d$ is at most exponential in $n$.

The idea of the proof of this estimate is to examine the restrictions placed on a Levi decomposition $G=R \cdot S$ by the normalizer fibration $X \rightarrow Y$. The base $Y$ is homogeneous under the semisimple factor $S$ and can be equivariantly embedded in projective space, thus providing the estimate $\operatorname{dim} S \leq m(m+2)$ where $m=\operatorname{dim} Y$. The group $N$ that acts transitively on the fiber normalizes $H^{0}$, contains the radical $R$ of $G$, and has a unimodular quotient, $N / H^{0}$. These facts have strong consequences for the weights of the adjoint representation of $S$ on the Lie algebra of $R$. The highest weights of this representation are shown to be bounded above (coordinate-wise) by the weight $\mu_{Y}$ associated to the anticanonical bundle of $Y, K_{Y}^{*}=\operatorname{det} T_{Y}$. The result of [8] provides an upper bound for the dimension of the irreducible representation $V\left(\mu_{Y}\right)$ with highest weight $\mu_{Y}$ : $\operatorname{dim} V\left(\mu_{Y}\right)=\operatorname{dim} H^{0}\left(Y, K_{Y}^{*}\right) \leq\left(\begin{array}{c}2 m+1 \\ m\end{array}\right)$. This estimate then leads to an upper bound for $\operatorname{dim} R$ which, along with the estimate for $\operatorname{dim} S$, yields the given upper bound for $d$.

\section{Preliminaries}

As in the introduction, let $X=G / H$ be a connected compact complex manifold homogeneous with respect to $G=\operatorname{Aut}^{0}(X)$. Let $G=R \cdot S$ be a Levi decomposition of $G$ into its radical $R$ and a semisimple complex Lie group $S$. Let $N=N_{G}\left(H^{0}\right)$ be the normalizer in $G$ of the connected component of the identity of $H$. Let $Y=G / N$ be the base of the normalizer fibration, $G / H \rightarrow G / N$. Then $Y=S / P$, where $P=S \cap H$ is a parabolic subgroup of $S$. The fiber $Z=N / H=\left(N / H^{0}\right) /\left(H / H^{0}\right)$ is the quotient of a complex Lie group by a uniform discrete subgroup; see [9], 3].

We use German letters, $\mathfrak{g}, \mathfrak{h}$, etc., to denote the Lie algebras of the Lie groups $G, H$, etc.

Let $T$ be a maximal torus of $S$. Let $\Phi \subset \mathfrak{t}^{*}$ denote the roots of $S$ with respect to $T$ and let $\left\{\alpha_{1}, \ldots, \alpha_{\ell}\right\} \subset \Phi$ be a system of simple roots. Let $\Phi^{+}$denote the subset of positive roots, i.e., those that are positive integral combinations of the simple roots. The negative roots are denoted by $\Phi^{-}=-\Phi^{+}$. For any root $\alpha \in \Phi$, let $e_{\alpha} \in \mathfrak{s}$ be the corresponding root vector, $\left[x, e_{\alpha}\right]=\alpha(x) e_{\alpha}$ for all $x \in \mathfrak{t}$. We let $B$ denote the Borel subgroup of $S$ generated by $T$ and the negative root groups $\exp \mathbb{C} e_{\alpha}$, for all $\alpha \in \Phi^{-}$. We may assume that $P$ contains $B$.

Let $\lambda_{1}, \ldots, \lambda_{\ell}$ be the fundamental dominant weights of $S$ defined by $\left\langle\lambda_{i}, \alpha_{j}\right\rangle=$ $2\left(\lambda_{i}, \alpha_{j}\right) /\left(\alpha_{j}, \alpha_{j}\right)=\delta_{i j}$ where $($,$) denotes the Killing form. Any weight \mu \in \mathfrak{t}^{*}$ can be written $\mu=\sum_{i=1}^{\ell}\left\langle\mu, \alpha_{i}\right\rangle \lambda_{i}$. The irreducible representation of $S$ with highest 
weight $\mu$ is denoted by $V(\mu)$. If $V$ is a finite-dimensional $T$-module we denote by $\chi(V) \in \mathfrak{t}^{*}$ the character of $V$, i.e., the sum of the weights of $V$.

Let $P=R_{P} \cdot S_{P}$ be a Levi decomposition of the parabolic subgroup $P$. We let $\Phi_{P}$ denote the subsystem of roots of $S_{P}$ and let $\Phi_{P}^{+}=\Phi_{P} \cap \Phi^{+}$. Let $I$ denote the subset of indexes, $I \subset\{1, \ldots, \ell\}$, such that $\Phi_{P}^{+} \cap\left\{\alpha_{1}, \ldots, \alpha_{\ell}\right\}=\left\{\alpha_{i}\right\}_{i \in I}$. The conjugacy class of $P$ is uniquely determined by $I$ and any such choice of indexes is associated to a parabolic subgroup of $S$.

We define $\Phi_{Y}^{+}=\Phi^{+} \backslash \Phi_{P}^{+}$. These roots clearly coincide with the negatives of the roots of $R_{P}$. Since $T_{Y}$ is generated at the identity coset by the root vectors $e_{\alpha} \in \mathfrak{s}$ for $\alpha \in \Phi_{Y}^{+}$, the anticanonical bundle $K_{Y}^{*}=\bigwedge^{m} T_{Y}, m=\operatorname{dim} Y$, is the homogeneous line bundle associated to the weight

$$
\mu_{Y}=\sum_{\alpha \in \Phi_{Y}^{+}} \alpha=-\chi\left(\mathfrak{r}_{P}\right) .
$$

The weight $\mu_{Y}$ is dominant: $\left\langle\mu_{Y}, \alpha_{i}\right\rangle>0$ for $i \notin I$, and $\left\langle\mu_{Y}, \alpha_{i}\right\rangle=0$ for $i \in I$. In particular, $K_{Y}^{*}$ is a very ample line bundle and $\mu_{Y}$ is orthogonal to the roots $\Phi_{P}^{+}$. A simple formula for determining the coefficients $\left\langle\mu_{Y}, \alpha_{i}\right\rangle$ can be found in [6]. An important component in the proof of the main theorem is the following estimate on $\operatorname{dim} V\left(\mu_{Y}\right)=\operatorname{dim} H^{0}\left(Y, K_{Y}^{*}\right)$.

Theorem 1 ([8]). Let $Y$ be a homogeneous projective rational manifold of dimension $m$. Then

$$
3^{m} \leq \operatorname{dim} H^{0}\left(Y, K_{Y}^{*}\right) \leq\left(\begin{array}{c}
2 m+1 \\
m
\end{array}\right)
$$

with equality in the lower bound if and only if $Y$ is a flag manifold and equality in the upper bound if and only if $Y$ is complex projective space.

\section{A BOUnd For $\operatorname{dim} \operatorname{Aut}(X)$}

We retain the notation of the previous section. In particular, $X=G / H$ is a homogeneous compact complex manifold; $G=R \cdot S$ is a Levi decomposition with $R$ the radical of $G$ and $S$ semisimple; $Y=G / N=S / P$ is the base of the normalizer fibration $G / H \rightarrow G / N ; N=N_{G}\left(H^{0}\right)=R \cdot P ; P$ is a parabolic subgroup of $S$ with Levi decomposition $P=R_{P} \cdot S_{P} ; I$ is the set of indexes that give the generators $\left\{\alpha_{i}\right\}_{i \in I}$ of the positive roots of $S_{P} ; \mu_{Y}$ denotes the weight of the anticanonical bundle $K_{Y}^{*}$ of $Y$, and $\chi(V)$ denotes the character of an $S$-module $V$. The following lemma describes a well-known property of $\mathfrak{s l}_{2}$-modules (see, e.g., [1, p. 90]).

Lemma 1. Let $V$ be a finite-dimensional $\mathfrak{s l}_{2}$-module, and let $W$ be a proper subspace of $V$ consisting of weight spaces. Assume that $W$ is invariant under $e_{-\alpha}$ where $\alpha$ is a positive root. Then for any highest weight $\lambda$ of $V$,

$$
0 \leq \lambda\left(x_{\alpha}\right) \leq \chi(V / W)\left(x_{\alpha}\right)
$$

Proof. Let $\lambda_{1}=\lambda$, and let $V=V\left(\lambda_{1}\right) \oplus \cdots \oplus V\left(\lambda_{t}\right)$ be a decomposition of $V$ into irreducible representations with highest weights $\lambda_{1}, \ldots, \lambda_{t}$. Let $W=W_{1} \oplus \cdots \oplus W_{t}$, $W_{i}=W \cap V\left(\lambda_{i}\right), 1 \leq i \leq t$, be the corresponding decomposition of $W$. Since $W_{i}$ is invariant under $e_{-\alpha}$, there is a nonnegative integer $k_{i}$ such that the weights of $V\left(\lambda_{i}\right) / W_{i}$ are $\lambda_{i}, \lambda_{i}-\alpha, \ldots, \lambda_{i}-k_{i} \alpha$. Cancelling any negative terms with corresponding positive terms we find there is a nonnegative integer $k_{i}^{\prime} \leq k_{i}$ such that

$$
\chi\left(V\left(\lambda_{i}\right) / W_{i}\right)\left(x_{\alpha}\right)=\lambda_{i}\left(x_{\alpha}\right)+\left(\lambda_{i}\left(x_{\alpha}\right)-2\right)+\cdots+\left(\lambda_{i}\left(x_{\alpha}\right)-2 k_{i}^{\prime}\right)
$$


with each term $\lambda_{i}\left(x_{\alpha}\right)-2 j \geq 0$ for $j=0, \ldots, k_{i}^{\prime}$. Therefore,

$$
\chi(V / W)\left(x_{\alpha}\right)=\sum_{i=1}^{t} \chi\left(V\left(\lambda_{i}\right) / W_{i}\right)\left(x_{\alpha}\right) \geq \chi\left(V\left(\lambda_{1}\right) / W_{1}\right) \geq \lambda_{1}\left(x_{\alpha}\right) \geq 0 .
$$

The next proposition provides crucial information about the weights of the representation of $S$ on $\mathfrak{r}$.

\section{Proposition 1.}

a) $\chi(\mathfrak{h})=-\mu_{Y}$.

b) $\chi(\mathfrak{r} / \mathfrak{r} \cap \mathfrak{h})=\mu_{Y}+\chi\left(\mathfrak{r}_{P} \cap \mathfrak{h}\right)$.

c) $\chi(\mathfrak{r} / \mathfrak{r} \cap \mathfrak{h})$ is a dominant weight whose coefficients satisfy $\left\langle\chi(\mathfrak{r} / \mathfrak{r} \cap \mathfrak{h}), \alpha_{i}\right\rangle=0$ for $i \in I$ and $0 \leq\left\langle\chi(\mathfrak{r} / \mathfrak{r} \cap \mathfrak{h}), \alpha_{i}\right\rangle \leq\left\langle\mu_{Y}, \alpha_{i}\right\rangle$ for $i \notin I$.

Proof. a) Since $H / H^{0}$ is a uniform discrete subgroup of $N / H^{0}$, the latter group is unimodular (see, e.g., [5]). Thus, $\chi(\mathfrak{n} / \mathfrak{h})=0$ and $\chi(\mathfrak{h})=\chi(\mathfrak{n})$. Since $\mathfrak{n}=\mathfrak{r}+\mathfrak{r}_{P}+\mathfrak{s}_{P}$ with $\chi(\mathfrak{r})=0\left(\mathfrak{r}\right.$ is an $S$-module), $\chi\left(\mathfrak{s}_{P}\right)=0$, and $\chi\left(\mathfrak{r}_{P}\right)=-\mu_{Y}$ by (1), we see that $\chi(\mathfrak{h})=-\mu_{Y}($ compare with Lemma 1 in [1, p. 96]).

b) On the other hand, $\chi(\mathfrak{h})=\chi(\mathfrak{r} \cap \mathfrak{h})+\chi\left(\mathfrak{r}_{P} \cap \mathfrak{h}\right)+\chi\left(\mathfrak{s}_{P} \cap \mathfrak{h}\right)$. Since $\mathfrak{s}_{P} \cap \mathfrak{h}$ is an ideal in $\mathfrak{s}_{P}$, it is either trivial or equals $\mathfrak{s}_{P}$, and hence its character is zero. Therefore, using a), $\chi(\mathfrak{r} / \mathfrak{r} \cap \mathfrak{h})=-\chi(\mathfrak{r} \cap \mathfrak{h})=-\chi(\mathfrak{h})+\chi\left(\mathfrak{r}_{P} \cap \mathfrak{h}\right)=\mu_{Y}+\chi\left(\mathfrak{r}_{\mathfrak{p}} \cap \mathfrak{h}\right)$.

c) Because $\mathfrak{r}_{P} \cap \mathfrak{h}$ is invariant under $\mathfrak{s}_{P}, \chi\left(\mathfrak{r}_{P} \cap \mathfrak{h}\right)\left(x_{\alpha_{i}}\right)=0$ for $i \in I$, and therefore $\chi(\mathfrak{r} / \mathfrak{r} \cap \mathfrak{h})\left(\alpha_{i}\right)=0$ for $i \in I$. To see that $\chi(\mathfrak{r} / \mathfrak{r} \cap \mathfrak{h})$ is dominant, let $\alpha$ be any positive root and let $s_{\alpha}$ be the Lie algebra isomorphic to $\mathfrak{s l}_{2}$ generated by $e_{\alpha}, e_{-\alpha}$ and $x_{\alpha}=\left[e_{\alpha}, e_{-\alpha}\right]$. Since $\mathfrak{r} \cap \mathfrak{h}$ is invariant under $e_{-\alpha}$, we obtain from Lemma 1 that $\chi(\mathfrak{r} / \mathfrak{r} \cap \mathfrak{h})\left(x_{\alpha}\right) \geq 0$, and hence $\chi(\mathfrak{r} / \mathfrak{r} \cap \mathfrak{h})$ is dominant.

It remains to prove that $\chi\left(\mathfrak{r}_{P} \cap \mathfrak{h}\right)$ is a negative dominant weight, i.e., that $\left\langle\chi\left(\mathfrak{r}_{P} \cap \mathfrak{h}\right), \alpha_{i}\right\rangle \leq 0$ for $i \notin I$. Let $J=\left\{\beta_{1}, \ldots, \beta_{m}\right\}$ be the set of weights of $\mathfrak{r}_{P} \cap \mathfrak{h}$. Then $\chi\left(\mathfrak{r}_{P} \cap \mathfrak{h}\right)$ is the weight of $x_{\beta_{1}} \wedge \cdots \wedge x_{\beta_{m}} \in \wedge^{m} \mathfrak{s}$. For any negative root $\alpha$, $\mathfrak{r}_{P} \cap \mathfrak{h}$ is invariant under $x_{\alpha}$. Thus, if $\beta_{i}+\alpha$ is a root, then $\beta_{i}+\alpha \in J$, so that

$$
x_{\alpha} \cdot x_{\beta_{1}} \wedge \cdots \wedge x_{\beta_{m}}=\sum_{i=1}^{m} c_{i} x_{\beta_{1}} \wedge \cdots \wedge x_{\beta_{i}+\alpha} \wedge \cdots \wedge x_{\beta_{m}}=0 .
$$

Therefore, $x_{\beta_{1}} \wedge \cdots \wedge x_{\beta_{m}}$ is a lowest weight vector in $\wedge^{m} \mathfrak{s}$ and $\chi\left(\mathfrak{r}_{P} \cap \mathfrak{h}\right)$ must be negative dominant.

Theorem 2. Let $X$ be a homogeneous compact complex manifold of dimension $n \geq$ 3. Then

$$
\operatorname{dim} \operatorname{Aut}(X) \leq n^{2}-1+\left(\begin{array}{c}
2 n-1 \\
n-1
\end{array}\right) .
$$

Proof. Let $m=\operatorname{dim} Y$ and $p=\operatorname{dim} Z=n-m$. Since $S$ acts transitively on $Y$, $\operatorname{dim} S \leq m(m+2)$. To find an estimate for $\operatorname{dim} R$ we decompose $\mathfrak{r}$ into irreducible $S$-modules, $\mathfrak{r}=V\left(\lambda_{1}\right) \oplus \cdots \oplus V\left(\lambda_{t}\right)$. For any positive root $\alpha$, Lemma 1 and Proposition 1imply that $0 \leq\left\langle\lambda_{i}, \alpha\right\rangle=\lambda_{i}\left(x_{\alpha}\right) \leq \chi(\mathfrak{r} / \mathfrak{r} \cap \mathfrak{h})\left(x_{\alpha}\right)=\left\langle\chi(\mathfrak{r} / \mathfrak{r} \cap \mathfrak{h}), x_{\alpha}\right\rangle \leq\left\langle\mu_{Y}, \alpha\right\rangle$. So $\operatorname{dim} V\left(\lambda_{i}\right) \leq \operatorname{dim} V\left(\mu_{Y}\right)$, for $1 \leq i \leq t$, by the Weyl dimension formula [4]. Therefore, $\operatorname{dim} R=\sum_{i=1}^{t} \operatorname{dim} V\left(\lambda_{i}\right) \leq t \operatorname{dim} V\left(\mu_{Y}\right)$. The number of irreducible components, $t$, cannot exceed $p=\operatorname{dim} N / H$ : If $t>p$, then $V\left(\lambda_{i}\right) \subset \mathfrak{h}$ for at least one $i$ (since $N=R \cdot P$ ) and the ideal generated by $V\left(\lambda_{i}\right)$ in $\mathfrak{r}$ would be an ideal 
of $\mathfrak{g}$ contained in $\mathfrak{h}$, contradicting the fact that $G$ acts effectively on $X=G / H$ (compare with Lemma 2 in [1, p. 97]). We conclude that $\operatorname{dim} R \leq p \operatorname{dim} V\left(\mu_{Y}\right)$.

Applying Theorem 1, we arrive at the estimate

$$
\operatorname{dim} G=\operatorname{dim} S+\operatorname{dim} R \leq m(m+2)+(n-m)\left(\begin{array}{c}
2 m+1 \\
m
\end{array}\right) .
$$

If $m=0$, then $\operatorname{dim} G=\operatorname{dim} R=n$ and if $m=n$, then $\operatorname{dim} G=\operatorname{dim} S \leq n(n+2)$. For $n \geq 3$ and $1 \leq m \leq n-1$, the maximum of the right-hand side of (2) occurs for $m=n-1$, and this maximum always exceeds $n(n+2)$. Therefore,

$$
\operatorname{dim} G \leq(n-1)(n+1)+\left(\begin{array}{c}
2(n-1)+1 \\
n-1
\end{array}\right) .
$$

\section{REFERENCES}

[1] Akhiezer, D., Lie Group Actions in Complex Analysis, Vieweg, Braunschweig, 1995. MR 96g:32051

[2] Bochner, S. and Montgomery, D., Groups on analytic manifolds, Ann. Math. 48 (1947), 659-669. MR 9:174f

[3] Borel, A. and Remmert, R., Über kompakte homogene Kählersche Mannigfaltigkeiten, Math. Ann. 145 (1961/1962), 429-439. MR 26:3088

[4] Fulton, W. and Harris, J., Representation Theory, Springer-Verlag, Berlin, Heidelberg, New York, 1991. MR 93a:20069

[5] Raghanuthan, M. S., Discrete Subgroups of Lie Groups, Springer-Verlag, New York, 1972. MR 58:22394a

[6] Snow, D., The nef value of homogeneous line bundles and related vanishing theorems, Forum Math. 7 (1995), 385-392. MR 96a:14057

[7] Snow, D. and Winkelmann, J., Compact complex homogeneous manifolds with large automorphism groups, Invent. Math. 134 (1998), 139-144. MR 99f:32054

[8] Snow, D., Bounds for the anticanonical bundle of a homogeneous projective rational manifold, preprint (http://www.nd.edu/ ${ }^{\sim}$ snow).

[9] Tits, J., Espaces homogènes complexes compacts, Comment. Math. Helv. 37 (1962/1963), 111-120. MR 27:4248

Department of Mathematics, University of Notre Dame, Notre Dame, Indiana 46556

E-mail address: snow.1@nd.edu 